\title{
THE CRITICAL POINTS OF A TYPICALLY-REAL FUNCTION
}

\author{
A. W. GOODMAN ${ }^{1}$
}

ABstract. The critical points of a typically-real function cannot lie too close to the real axis. By adding a mild restriction, we determine $D_{k}$ the domain of variability of a $k$ th order critical point. Similar results are obtained for a $k$ th order branch point. We determine the domain of univalence for typically-real functions and propose a reasonable conjecture for the domain of $k$-valence.

1. Introduction. A function

$$
f(z)=z+\sum_{n=2}^{\infty} a_{n} z^{n},
$$

regular in $E:|z|<1$, is said to be typically-real if it satisfies the condition

$$
(\mathfrak{I}(z))(\mathfrak{I} z)>0
$$

for all nonreal $z$ in $E$. This class of functions (which we denote by TR) was introduced by Rogosinski [9] in 1932 and has been the object of many investigations ([2], [3], [7]).

The condition (2) implies that $f(z)$ is real in $E$, if and only if $z$ is real. Further it implies that if $-1<z<1$, then $f^{\prime}(z)>0$. It is intuitively obvious that if $c$ is a critical point of $f(z)$, a point where $f^{\prime}(z)=0$, then $c$ cannot lie too close to the real axis. In this work we determine this forbidden domain precisely. More generally, if $c_{k}$ is a critical point of $k$ th order, it cannot lie too close to the real axis. In this case we advance the conjecture that a certain domain $D_{k}$ is the forbidden domain. If we add a suitable condition on $f(z)$, then we can prove that $c_{k} \notin D_{k}$. We also obtain similar results about the location of a branch point $b_{k}=f\left(c_{k}\right)$ when $f(z) \in \mathrm{TR}$. The work closes with a theorem on the domain of univalence of the class TR and a conjecture on the domain of $k$-valence of the class TR. Our main tool is the theory of subordination, but we also use a Stieltjes integral representation due to M. S. Robertson [7].

Received by the editors June 20, 1972.

AMS (MOS) subject classifications (1970). Primary 30A24, 30A32, 30A36; Secondary 30A04, 30A42.

Key words and phrases. Typically-real functions, critical points, branch points, subordination, Schwarz lemma, univalent functions, multivalent functions.

${ }^{1}$ This research was completed while the author held a Faculty Development Leave Award granted by the University of South Florida.

(C) American Mathematical Society 1973 
2. The example function. In any subordination argument we need an example function which is a superordinate function for the problem under consideration. Let $\hat{S}_{k}$ be the surface obtained by attaching to a base plane $k$ half planes $\Im w>0$ at a branch point $B_{k}=\left|B_{k}\right| i$; and $k$ half planes $\mathfrak{I}_{w}<0$ at $\bar{B}_{k}$, where $B_{k}$ is yet to be determined. Let $F_{k}(z)$ be the function in the class TR that maps $E$ onto the surface $\hat{S}_{k}$. We will obtain $F_{k}(z)$ explicitly by a sequence of transformations. We consider first the half disk $E^{(l)} \equiv$ $E \cap\{z \mid \Re z<0\}$. The linear transformation $u=-(1+z i) /(i+z)$ maps $E^{(l)}$ onto the first quadrant; then $v=u^{2}$ carries this quadrant onto the upper half plane, and finally $w=-i(v-i) /(v+i)$ takes this half plane onto $E$. The composition of these mappings gives

$$
T_{1}(z) \equiv \frac{1+2 z-z^{2}}{1-2 z-z^{2}}
$$

a function that carries $E^{(l)}$ onto $E$. It is important to note that: $T_{1}(0)=1$, $T_{1}(i)=i, T_{1}(-1)=-1$, and $T_{1}(-i)=-i$. Hence the diameter from $-i$ to $i$ is mapped onto the arc $-i, 1, i$ of the boundary of $E$.

Next, with $k$ a fixed natural number, let $\eta=e^{\pi i /(k+1)}$, and let

$$
T_{2}(z)=(z+s) /(1+s z)
$$

where $s$ is real and adjusted so that $T_{2}$ maps the arc $-i, 1, i$ onto the arc $\bar{\eta}, 1, \eta$. A brief computation gives

$$
s=\frac{\cos (\pi /(k+1))}{1+\sin (\pi /(k+1))} .
$$

The function

$$
T_{3}(z)=\left(1-z^{k+1}\right) /\left(1+z^{k+1}\right)
$$

carries $E$ onto a domain consisting of $k+1$ half planes $\Re w>0$. It is worth noting that $T_{3}$ maps the arc $\bar{\eta}, 1, \eta$ onto the imaginary axis.

Finally we define $F_{k}(z)$ by

$$
F_{k}(z) \equiv \frac{i}{2(k+1) A} T_{3}\left(T_{2}\left(T_{1}(i z)\right)\right)
$$

where

$$
A=(1-s) /(1+s) \text {. }
$$

By our construction $F_{k}(z)$ maps the half disk $E^{(+)} \equiv E \cap\{z \mid \mathfrak{I} z>0\}$ onto the surface formed by $k+1$ half planes $\Im w>0$, tied together with a $k$ th order branch point. If we reflect the half disk across the real axis, and the image domain across the real axis, we find that $F_{k}(z)$ maps $E$ onto $\hat{S}_{k}$ as required. 
A computation shows that

$$
F_{k}(z)=\frac{i}{2(k+1) A} \frac{P^{k+1}(z)-Q^{k+1}(z)}{P^{k+1}(z)+Q^{k+1}(z)},
$$

where

$$
P(z) \equiv 1-2 A i z+z^{2}, \quad Q(z) \equiv 1+2 A i z+z^{2} .
$$

The factor $1 / 2(k+1) A$ is selected so that $F_{k}^{\prime}(0)=1$. We have

LemMa 1. For each positive integer $k$, the function $F_{k}(z)$, defined by equations (5), (8), (9), and (10), maps $E$ onto the surface $\hat{S}_{k}$. Further $F_{k}(z) \in \mathrm{TR}$. $F_{k}(z)$ has two critical points of $k$ th order, one at

$$
C_{k} \equiv R_{k} i \equiv\left(\left(A^{2}+1\right)^{1 / 2}-A\right) i,
$$

and the other at $-C_{k}$. The corresponding branch points are at $\pm i / 2(k+1) A \equiv$ $\pm B_{k}$.

In particular, $k=1,2$ gives

$$
F_{1}(z)=\frac{z\left(1+z^{2}\right)}{\left(1-z^{2}\right)^{2}}, \quad R_{1}=\sqrt{ } 2-1, \quad B_{1}=i / 4,
$$

and

$$
F_{2}(z)=\frac{z\left(9+14 z^{2}+9 z^{4}\right)}{9\left(1-z^{2}\right)^{2}\left(1+z^{2}\right)}, \quad R_{2}=\sqrt{ } 3 / 3, \quad B_{2}=i / 2 \sqrt{ } 3 .
$$

3. The critical points. Let $D_{k}$ be the domain that is bounded above by the arc of the circle through the points $-1, R_{k} i$, and 1 , and bounded below by the arc of the circle through $-1,-R_{k} i$ and 1 . Here $R_{k}$ is defined by equations (5), (8) and (11). Then we have

THEOREM 1. Suppose that $f(z) \in \mathrm{TR}$ and that $f(z)$ has a kth order critical point at $c_{k}$. Suppose further that $f\left(c_{k}\right)=b_{k}$, and that the equation $f(z)=b_{k}$ has no solution in $E$, except $z=c_{k}$. Then the point $c_{k}$ must lie in $E-D_{k}$. Further, for each $c_{k}$ in $E-D_{k}$ there is an $f(z) \in \mathrm{TR}$ that has a kth order critical point at $c_{k}$. If $c_{k}$ is on the boundary of $D_{k}$ and $c_{k} \neq \pm 1$, then $f(z)$ is unique.

Proof. Since each function in TR has real coefficients, the image of $E$ is symmetric with respect to the real axis. Hence we can restrict our attention to critical points that lie in $E^{(+)}$and branch points that lie in the upper half plane. If $f(z) \in \mathrm{TR}$ and $r$ is in $(-1,1)$, then

$$
\phi(z) \equiv\left(f\left(\frac{z+r}{1+r z}\right)-f(r)\right) /\left(1-r^{2}\right) f^{\prime}(r)
$$


is also in TR. Since $(z+r) /(1+r z)$ moves the critical point along an arc of a circle through the points -1 and 1 , we can select $r$ so that $\phi(z)$ has its corresponding critical point on the imaginary axis. Hence without loss of generality we may assume that $\Re c_{k}=0$ and $\mathfrak{I} c_{k}>0$. Consequently it will be sufficient to prove that $\left|c_{k}\right| \geqq\left|C_{k}\right| \equiv R_{k}$. Next, we observe that if $f(z) \in \mathrm{TR}$, then $-f(-z)$ is also in TR. Hence if $b_{k}=\alpha+i \beta$ is the corresponding branch point we may assume that $\alpha \geqq 0$, and $\beta>0$.

We now adjust our example function $F_{k}(z)$ so that it has the same branch point. Since $F_{k}(z)$ maps $(-1,1)$ onto the real axis there is a real $t$ such that $F_{k}(t)=\alpha\left|B_{k}\right| / \beta$. Further, we set $a=\beta /\left|B_{k}\right|>0$. Then a brief computation shows that

$$
H(z) \equiv a\left[F_{k}\left(\frac{z-t}{1-t z}\right)+F_{k}(t)\right]
$$

has a $k$ th order critical point at $C_{k}^{\star}=\left(C_{k}+t\right) /\left(1+t C_{k}\right)$ and that $H\left(C_{k}^{\star}\right)=$ $\alpha+i \beta=b_{k}$. Since $F_{k}(z)$ is an odd function, we also have $H(0)=0$. If $H(E)=$ $S^{\star}$, then $S^{\star}$ is merely a translation followed by an expansion (or a contraction) of $\hat{S}$.

Let $z=G(w)$ be the inverse function of $H(z)$ defined on $S^{\star}$. Let us assume for the moment that the composite function $J(z) \equiv G(f(z))$ is well-defined. If so, it satisfies the conditions of Schwarz's lemma because $f(z)$ maps $E$ onto a surface $S$ that is "carried" by $S^{\star}$, and $G$ takes $S^{\star}$ onto $E$. Further, $J(0)=G(f(0))=G(0)=0$. Consequently for any $z$ in $E,|J(z)| \leqq|z|$, and because of our normalization the equality sign holds if and only if $J(z) \equiv z$. Now $J\left(c_{k}\right)=G\left(f\left(c_{k}\right)\right)=G\left(B_{k}\right)=\left(C_{k}+t\right) /\left(1+t C_{k}\right)$. Hence

$$
\left|c_{k}\right| \geqq\left|\left(R_{k} i+t\right) /\left(1+t R_{k} i\right)\right| \geqq R_{k},
$$

with equality only if $t=0$.

It remains to show that $J(z)$ is well-defined. This is the case if $f(z)$ is subordinate to $H(z)$. The concept of subordination was first used by Lindelöf [4], but the terminology was suggested by Littlewood [5]. The method was extensively exploited by Littlewood [5], Nehari [6], Rogosinski ([8], [10], [11], [12]), and others [2]. In most of the applications the superordinate function is either univalent or locally univalent. Whenever critical points occur in $H(z)$, the problem is avoided by adopting an alternate definition: $f(z)$ is subordinate to $H(z)$ if there is a $J(z)$ such that $f(z)=H(J(z))$ and $J(z)$ satisfies the conditions of Schwarz's lemma. Littlewood [5], and Beckenbach and Graham [1] give the following geometric criterion for $f(z)$ to be subordinate to $H(z)$.

Let $f(E)=S$ and let $H(E)=S^{\star}$. If there is a mapping of $S$ into $S^{\star}$ such that each point $w$ of $S$ goes into a point of $S^{\star}$ with the same complex coordinate, and each closed contour on $S$ beginning and ending at $f(0)$ 
goes into a closed contour of $S^{\star}$ beginning and ending at $H(0)$, then $f(z)$ is subordinate to $H(z)$, and the representation $f(z)=H(J(z))$ is indeed possible.

A mapping of the type just described will be called an $S^{\star}$-projection. In our problem $f(0)=H(0)=0$ and both functions have no other zeros in $E$. Both functions are univalent in a neighborhood of $z=0$, and hence there is an $S^{\star}$-projection in a neighborhood of $w=0$. Let $\gamma_{0}$ be the radial segment from $z=0$ to $z=c_{k}$ and let $\Gamma_{0}=f\left(\gamma_{0}\right)$. Since the inverse of $H(z)$ is locally univalent except for the point $w=b_{k}=B_{k}$, there is an $S^{\star}$-projection of $\Gamma_{0}$ onto a curve $\Gamma_{0}^{\star}$ of $S^{\star}$.

If $\gamma$ is any radial segment that does not pass through $z=c_{k}$ or $z=\bar{c}_{k}$ and $\Gamma=f(\gamma)$, then the $S^{\star}$-projection of $\Gamma$ established near $w=0$ can be continued over all of $\Gamma$ because $f(z) \neq b_{k}, \bar{b}_{k}$ on $\Gamma$, and $S^{\star}$ is locally univalent everywhere except at $b_{k}$ and $\bar{b}_{k}$. Finally consider the points $w=f(z)$ where $z$ is on the extension of the radial segment to $c_{k}$. Since every simple closed curve that encloses $c_{k}$ (and not $\bar{c}_{k}$ ) has an image under $f(z)$ that winds around $b_{k}, k$ times, and the same is true of the image under $H(z)$, the $S^{\star}$ projection can be extended to the remaining points of $S$. It is clear that the correspondence just described satisfies the Littlewood requirements, and hence $f(z)$ is subordinate to $H(z)$.

To establish an $S^{\star}$-projection it was necessary to assume that $f(z)=b_{k}$ has no solution in $E$ other than $z=c_{k}$. We conjecture that Theorem 1 is true without this hypothesis. A proof for the case $k=1$ will be given in $\S 5$.

4. The branch points. Let $F_{k}(z)$ be the extremal function defined in $\S 2$, and for each real $t$ in $(-1,1)$, set

$$
F_{k}(z, t)=\left(F_{k}\left(\frac{z-t}{1-t z}\right)+F_{k}(t)\right) / F_{k}^{\prime}(t)\left(1-t^{2}\right) .
$$

The function $F_{k}(z, t)$ has one critical point on the upper boundary of $D_{k}$, and as $t$ varies, this critical point (together with its conjugate) describes the boundary of $D_{k}$ (except for the points \pm 1 ). The corresponding branch points describe two curves, $\Gamma_{k}^{(+)}(w)$ in the upper half plane, and $\Gamma_{k}^{(-)}(w)$ in the lower half plane. Since $F_{k}\left(C_{k}\right)=i / 2(k+1) A \equiv B_{k}$ the curve $\Gamma_{k}^{(+)}(w)$ has the parametric representation $w=\left(F_{k}(t)+\left|B_{k}\right| i\right) /\left(F_{k}^{\prime}(t)\left(1-t^{2}\right)\right)$. A brief computation will show that for each fixed $k$, the curve $\Gamma_{k}^{(+)}(w)$ is starlike with respect to the origin and that as $t \rightarrow \pm 1, w \rightarrow \pm \frac{1}{4}$.

Let $D_{k}(w)$ be the union of the real axis and the domain bounded above by $\Gamma_{k}^{(+)}(w)$ and below by $\Gamma_{k}^{(-)}(w)$. With this notation we have

THEOREM 2. Let $f(z)$ satisfy the conditions of Theorem 1 . Then $b_{k} \notin D_{k}(w)$. For each point $b_{k}$ in the complement of $D_{k}(w)$, there is an $f(z) \in \mathrm{TR}$ with a 
$k$ th order branch point at $b_{k}$. If $b_{k}$ is on $\Gamma_{k}^{(+)}(w) \cup \Gamma_{k}^{(-)}(w)$ then there is only one such function.

Proof. As in $\S 3$, we select $a$ and $t$ so that $f(z)$ and $H(z)$ have the same branch point at $b_{k}$. If $G(w)$ is the inverse function of $H(z)$ on $S^{\star}$, then $J(z) \equiv$ $G(f(z))$ satisfies the conditions of Schwarz's lemma. Consequently $0<$ $J^{\prime}(0) \leqq 1$, and equality occurs if and only if $J(z) \equiv z$. But

$$
J^{\prime}(0)=\frac{f^{\prime}(0)}{a F_{k}^{\prime}(-t)\left(1-t^{2}\right)}=\frac{1}{a F_{k}^{\prime}(t)\left(1-t^{2}\right)} \leqq 1 .
$$

Hence $a>1 / F_{k}^{\prime}(t)\left(1-t^{2}\right)$ and consequently $H(z)=\mu F_{k}(z, t)$ where $\mu \geqq 1$, with equality if and only if $f(z)=F_{k}(z, t)$. Therefore the branch point of $f(z)$ lies at the end point of a radial segment that terminates on or passes through $\Gamma_{k}^{(+)}(w) \cup \Gamma_{k}^{(-)}(w)$. Since each of these curves is starlike with respect to the origin, this completes the proof.

5. A remark on valence. W. E. Kirwan [3] has proved that the radius of univalence for the class TR is $\sqrt{ } 2-1$. Since $C_{1}=(\sqrt{ } 2-1) i$ and since the transformation (14) moves this critical point along the upper boundary of $C_{1}$, Kirwan's result will give Theorem 1 when $k=1$.

I am indebted to E. B. Saff for calling my attention to the paper by Kirwan. Saff also suggested that perhaps Kirwan's circle of univalence could be enlarged to include the domain $D_{1}$.

THEOREM 3. Let $f(z) \in \mathrm{TR}$. Then $f(z)$ is univalent in $D_{1}$ and $D_{1}$ is the maximal domain of univalence for the class TR.

Proof. By the symmetry we can restrict ourselves to the upper half disk $E^{(+)}$. Let $f\left(z_{1}\right)=f\left(z_{2}\right)$ with $z_{1}$ and $z_{2}$ in $E^{(+)}$. There is a minimal arc of a circle through \pm 1 on which univalence fails, and hence we can assume that $z_{1}$ and $z_{2}$ lie on this arc. By a transformation of the type (14) we may further assume that $z_{1}$ and $z_{2}$ are symmetric with respect to the $y$-axis. Let $z_{2}=x+i y, z_{1}=-x+i y$ where $x>0$ and $y>0$.

M. S. Robertson [7] proved that each function in TR has a Stieltjes integral representation

$$
f(z)=\frac{1}{\pi} \int_{0}^{\pi} \frac{z d \mu(\theta)}{1-2 z \cos \theta+z^{2}}, \quad \int_{0}^{\pi} d \mu(\theta)=\pi,
$$

where $\mu(\theta)$ is nondecreasing on $[0, \pi]$. Consequently if $f\left(z_{1}\right)=f\left(z_{2}\right)$ we 
have

$$
\begin{aligned}
0 & =\int_{0}^{\pi}\left(\frac{z_{2}}{1-2 z_{2} \cos \theta+z_{2}^{2}}-\frac{z_{1}}{1-2 z_{1} \cos \theta+z_{1}^{2}}\right) d \mu(\theta) \\
& =\int_{0}^{\pi} \frac{\left(z_{2}-z_{1}\right)\left(1-z_{1} z_{2}\right) d \mu(\theta)}{\text { Denominator }}
\end{aligned}
$$

Since $\left(z_{2}-z_{1}\right)\left(1-z_{1} z_{2}\right) \neq 0$ we can write that

$$
0=\int_{0}^{\pi} \frac{d \mu(\theta)}{\text { Den. }} .
$$

Now equation (20) is impossible if $\mathfrak{R}($ Den. $)>0$. But

$$
\begin{gathered}
\Re(\text { Den. })=1-2 x^{2}-6 y^{2}+\left(x^{2}+y^{2}\right)^{2}+4\left(x^{2}+y^{2}\right) \sin ^{2} \theta \\
\geqq 1-2 x^{2}-6 y^{2}+\left(x^{2}+y^{2}\right)^{2} . \\
\Re(\text { Den. }) \geqq\left(1-x^{2}-y^{2}-2 y\right)\left(1-x^{2}-y^{2}+2 y\right),
\end{gathered}
$$

with equality only if $\theta=0$, or $\pi(\mu(\theta)$ has jumps only at 0 or $\pi)$. Since the two factors on the right side of (21) will give equations for the circles that form the boundary of $D_{1}$ we see that $\mathfrak{R}($ Den. $)>0$ in $D_{1}$.

It is clear that if $f(z) \in \mathrm{TR}$, the image of $D_{1}$ under $f(z)$ need not be starlike with respect to $w=0$. However, it seems likely that the image will be convex in the direction of the imaginary axis.

It also seems likely that every $f(z) \in \mathrm{TR}$ is at most $k$-valent in $D_{k}$ for every natural number $k$. If so, then our example functions show that $D_{k}$ is the maximal domain of $k$-valence for the class TR.

\section{REFERENCES}

1. E. F. Beckenbach and E. W. Graham, On subordination in complex variable theory. Construction and applications of conformal maps, Proc. Sympos., National Bureau of Standard, Appl. Math. Ser., no. 18, U.S. Government Printing Office, Washington, D.C., 1952, pp. 247-254. MR 14, 632.

2. S. D. Bernardi, Bibliography of schlicht functions, Courant Institute of Mathematical Sciences, New York University, Technical Report \#NR 041-019, IMM 351, Office of Naval Research, New York; New York University, New York, 1966. MR 34 \#2849.

3. W. E. Kirwan, Extremal problems for the typically real functions, Amer. J. Math. 88 (1966), 942-954. MR 34 \#2853.

4. E. Lindelöf, Mémoire sur certaines inégalités dans la théorie des fonctions monogènes et sur quelques propriétés nouvelles de ces fonctions dans le voisinage d'un point singulier essentiel, Acta Soc. Sci. Fenn. 35 (1908), no. 7.

5. J. E. Littlewood, On inequalities in the theory of functions, Proc. London Math. Soc. (2) 23 (1925), 481-519.

6. Z. Nehari, On analytic functions possessing certain properties of univalence, Proc. London Math. Soc. (2) 50 (1948), 120-136. MR 9, 576. 
7. M. S. Robertson, On the coefficients of a typically-real function, Bull. Amer. Math. Soc. 41 (1935), 565-572.

8. W. Rogosinski, Über Bildschranken bei Potenzreihen und ihren Abschnitten, Math. Z. 17 (1923), 260-276.

9. —_ Über positive harmonische Entwicklungen und typisch-reelle Potenzreihen, Math. Z. 35 (1932), 93-121.

10. —_ Zum Majorantenprinzip der Funktionentheorie, Math. Z. 37 (1933), 210 236.

11. - On subordinate functions, Proc. Cambridge Philos. Soc. 35 (1939), 1-36.

12. - On the coefficients of subordinate functions, Proc. London Math. Soc. (2) 48 (1943), 48-82.

Department of Mathematics, University of South Florida, Tampa, Florida 33620 